\title{
A case of postprandial hypotension in an individual with cervical spinal cord injury: treatment with acarbose
}

\author{
Clara Farrehi ${ }^{1} \cdot$ Carlotta Pazzi $^{1} \cdot$ Michael Stillman $^{2}$
}

Received: 16 July 2019 / Revised: 1 August 2019 / Accepted: 19 August 2019

(c) International Spinal Cord Society 2019

\begin{abstract}
Introduction Postprandial hypotension ( $\mathrm{PPH})$ is a postmeal drop in systolic blood pressure that may or may not be symptomatic. While the etiologies of PPH are incompletely understood, it is thought to occur when glucose absorption causes increased splanchnic blood flow or "pooling" in people who lack sufficient compensatory responses to support their systemic blood pressure. Postprandial hypotension is well described in individuals with neurodegenerative diseases, yet only rarely in people living with spinal cord injury (SCI). Acarbose is an alpha-glucosidase inhibitor that treats PPH by slowing gastric transit time and reducing glucose uptake in the small intestine, hence decreasing superior mesenteric artery blood flow.

Case presentation A 62-year-old woman with long-standing cervical SCI presented with 5 years of worsening postprandial lightheadedness, visual "flashes", and neck pain. She had had multiple episodes of near and frank syncope and her prior medical team had initiated midodrine three times daily. We began treatment with acarbose, starting at $50 \mathrm{mg}$ with each meal and rapidly titrating to $100 \mathrm{mg}$ at mealtime. She noticed an immediate improvement in her symptoms and an attenuation of postmeal drops in both systolic and diastolic blood pressures.

Discussion To our knowledge, this is one of the first described cases of PPH among people living with SCI. Given the autonomic dysfunction that frequently accompanies higher-level of injuries, it is possible that many more people with SCI have this condition, whether or not it is symptomatic. Acarbose is one of the several established treatments for PPH, and proved effective and tolerable for our patient.
\end{abstract}

\section{Introduction}

Postprandial hypotension (PPH) is defined as a decrease in systolic blood pressure (SBP) of $>20 \mathrm{mmHg}$ within $2 \mathrm{~h}$ of the start of a meal, or a decrease to $<90 \mathrm{mmHg}$ when the pre-prandial blood pressure (BP) is $100 \mathrm{mmHg}$ or higher $[1,2]$. Though most people with PPH are asymptomatic, its clinical manifestations may include dizziness, nausea, visual disturbances, and confusion [1,2]. As a result of

These authors contributed equally: Clara Farrehi, Carlotta Pazzi

Michael Stillman

michael.stillman@jefferson.edu

1 Haverford College, Haverford, PA, USA

2 Internal Medicine and Rehabilitation Medicine, Sidney Kimmel Medical College of Thomas Jefferson University, 1100 Walnut Street, Suite 601, Philadelphia, PA 19107, USA coronary and cerebral hypoperfusion, PPH may result in angina, syncope, and in rare cases, stroke and/or death [1]. While PPH may be found in "healthy" older individuals, it is often described in people with diseases associated with autonomic dysregulation including diabetes mellitus, the Parkinsonian syndromes, Alzheimer's disease, dementia with lewy bodies, and pure autonomic failure [3]. A 2014 systematic review of PPH documented prevalence rates of at least $25-30 \%$ in most of these conditions [2].

The pathophysiology of PPH is not well understood, but it is thought to be multifactorial. When the small intestine is exposed to nutrients-particularly glucose and fatsplanchnic blood flow is increased. A variety of disease and age-related autonomic and neurohormonal alterations may leave certain people unable to compensate for this mesenteric "pooling," and, as a result, they develop PPH [2]. Recommended treatments for $\mathrm{PPH}$ range from the conservative to the pharmacologic. Eating smaller meals with fewer carbohydrates and drinking large volumes of water before meals may help to attenuate PPH-related symptoms. 
While there are data supporting the use of caffeine pills, DL-Threo-3,4-dihydroxyphenylserine, guar gum, and metformin, evidence is far stronger for treatment with octreotide and acarbose [4-7]. While the former inhibits splanchnic vasodilation, the latter reduces carbohydrate breakdown and gut absorption, decreasing mesenteric perfusion demand.

Spinal cord injury (SCI) - particularly above the sixth thoracic level-may be associated with the loss of supraspinal sympathetic input with resulting cardiovascular impairment and hypotension [8, 9]. However, PPH has been described only rarely in the setting of SCI [10-12]. Catz et al. first noted this association in 1992, reporting an individual with a T3 SCI and PPH that was successfully managed with mealtime oral caffeine [11]. In 2007, Catz et al. evaluated 21 individuals with motor complete SCI (11 cervical and 10 thoracic) for PPH, finding that only those with thoracic injuries sustained postmeal hypotension but that it was asymptomatic [12]. In 2017, Ishikawa et al. described a 66-year-old gentleman with chronic T4 SCI who was found to have awakening hypertension and $\mathrm{PPH}$ without an identifiable secondary cause [10]. He was admitted to an inpatient service and successfully treated with droxidopa, a norepinephrine precursor. In this manuscript, we report a case of PPH in a woman with SCI that was successfully managed in the outpatient setting with acarbose.

\section{Case}

A 62-year-old woman (PG) was seen in our internal medicine clinic to establish care. She had a medical history of C4 AIS C SCI due to a motor vehicle accident 35 years prior, intermittent hypertension, chronic bilateral shoulder pain, and chronic cholelithiasis with postprandial abdominal pain. Five years earlier, PG had noticed postprandial dizziness and lightheadedness that had gradually worsened. These episodes had been attributed by a consulting cardiologist to an "autonomic nervous system disorder" and she had been started on midodrine $2.5 \mathrm{mg}$ taken three times daily with meals. She had also been given pindolol $5 \mathrm{mg}$ each day in an attempt to normalize her blood pressure. Her other medications included Vitamin D3 1000 IU/day, Cranberry tablets $500 \mathrm{mg}$ three times daily, and Oxybutynin $5 \mathrm{mg}$ three times daily.

On physical examination, PG had a blood pressure of $160 / 96$ with a pulse of 68 beats per minute. She weighed 150 pounds and had an oxygen saturation of $98 \%$. She was a well-appearing woman with fluid speech, her lungs were clear to auscultation, and her cardiovascular examination was without the evidence of valvulopathy or bruits, though she had $1+$ bilateral pitting edema in her distal legs without
Table 1 Oral glucose tolerance test results

\begin{tabular}{lll}
\hline & $\begin{array}{l}\text { Glucose } \\
(\text { ref range-mg/dL) }\end{array}$ & $\begin{array}{l}\text { Insulin } \\
(\text { ref range-mlU/mL) }\end{array}$ \\
\hline Time 1 $(0 \mathrm{~h})$ & $83(\mathrm{nl}<100)$ & $13.8(2-19.6)$ \\
Time 2 $(30 \mathrm{~min})$ & 158 & $118.1(6-86)$ \\
Time 3 $(1 \mathrm{~h})$ & 222 & $945(8-112)$ \\
Time 4 $(2 \mathrm{~h})$ & $234(\mathrm{nl}<140)$ & $255.8(5-55)$ \\
\hline
\end{tabular}

diminished pedal pulses. Initial laboratory results revealed a mild anemia and normal electrolytes and kidney and liver function.

We referred PG for laparoscopic cholecystectomy. She had no postoperative complications and immediate resolution of her abdominal pain. An oral glucose tolerance test was performed, and PG was found to have diabetes mellitus (Table 1). Home blood pressure monitoring revealed large drops in systolic and diastolic blood pressures within $20 \mathrm{~min}$ of mealtime without compensatory elevations in heart rate. Even while taking midodrine, PG reported frequent lightheadedness, visual disturbances, neck pain, and having to "lean forward on a pillow" to ensure she did not pass out.

We asked PG to stop taking midodrine and to monitor her blood pressure and heart rate before and after three consecutive meals (Table 2). During that day, her average postprandial drop in SBP was over $47 \mathrm{mmHg}$. She started taking acarbose $50 \mathrm{mg}$ just prior to eating a meal, and within 1 week, the dose was increased to $100 \mathrm{mg}$ with meals. While PG continued to have PPH on the medication, both her postmeal hypotension and her symptoms were substantially ameliorated. She now experiences infrequent mild lightheadedness after eating, but has noted increased flatulence, a known side effect of acarbose [13]. Despite this, PG has not reported changes in bowel transit time or stool consistency.

\section{Discussion}

In this report, we describe a 62-year-old woman with longstanding cervical SCI and PPH whose symptoms and blood pressure drops were substantively improved with acarbose therapy, even though she continues to meet criteria for the condition.

PPH has only been described in several individuals with $\mathrm{SCI}$, though it is notable that during preparation of this manuscript, two people with SCI seen in our clinic complained of similar symptoms to PG's. We suspect that many people with higher-level SCI, who may have diminished ability to mount vasoconstrictive and tachycardic responses to hypotension, may suffer from PPH, and that their medical teams simply have not yet diagnosed or treated it. 
Table 2 Mean pre and postprandial blood pressure and heart rate: with and without acarbose

\begin{tabular}{|c|c|c|c|c|c|c|c|c|c|}
\hline & Premeal SBP & Postmeal SBP & Delta & Premeal DBP & Postmeal DPB & Delta & Premeal HR & Postmeal HR & Delta \\
\hline Before acarbose $(n=3)$ & 145 & 97.67 & 47.33 & 95.67 & 60 & 35.67 & 69.33 & 73.67 & +4.34 \\
\hline After acarbose $(n=8)$ & 151.38 & 127.25 & 24.13 & 95.13 & 80.86 & 14.27 & 76.75 & 78.36 & +1.61 \\
\hline
\end{tabular}

Bold values indicates differences between pre and post meal readings

The significance of PG's diagnosis of diabetes mellitus is uncertain. It is believed that between 28 and $56 \%$ of people with traumatic SCI are diagnosed with impaired glucose tolerance or DM during OGTT [14-17], yet their fasting glucose levels tend to be normal [14]. In our earlier work, we showed that a cohort of individuals with SCI had higher glucose and insulin levels than able-bodied controls during OGTT but that their glycohemoglobin (HbA1c) levels were no different, perhaps indicating isolated postprandial hyperglycemia interrupting a largely euglycemic state [18]. There is some evidence that postprandial insulin spikes decrease peripheral and splanchnic vascular resistance, thereby dropping blood pressure and increasing "pooling" $[19,20]$. PG was markedly hyperinsulinemic during OGTT, so this may have exacerbated her PPH. It is also known that PPH is associated with DM, and that between 37 and $44 \%$ of people with this condition may be diagnosed with it [21,22].

A mitigating factor in PG's PPH is her treatment with pindolol, a nonselective beta-adrenergic antagonist. Even though PG's dose is very low (maximum daily dose is $60 \mathrm{mg}$ ), it may blunt her ability to mount a heart rate response to PPH. She has on multiple occasions declined the opportunity to stop this medication or to replace it with an antihypertensive agent that will not affect her heart rate. In addition, we did not evaluate PG for other causes of postural hypotension such as valvulopathies, profound volume depletion, or adrenal insufficiency. Given that her initial physical examination and laboratory evaluation did not reveal evidence of these conditions, and that her blood pressure alterations and symptoms were exclusively post prandial, we felt that further work up would be unnecessary.

In conclusion, this case indicates that clinicians caring for individuals with SCI ought to be alert to the possibility of $\mathrm{PPH}$. While the condition may be asymptomatic, it may also cause significant symptoms or, in severe cases, be life threatening. In addition, while several lifestyle interventions and medications have been shown to be effective for the treatment of PPH, acarbose is a viable option for people with PPH and SCI, particularly if they have concomitant DM.

\section{Data availability}

All data generated or analyzed during this report are included in this published article.

\section{Compliance with ethical standards}

Conflict of interest The authors declare that they have no conflict of interest.

Publisher's note: Springer Nature remains neutral with regard to jurisdictional claims in published maps and institutional affiliations.

\section{References}

1. Pavelic A, Krbot Skoric M, Crnosija L, Habek M. Postprandial hypotension in neurological disorders: systematic review and meta-analysis. Clin Auton Res. 2017;27:263-71.

2. Trahair LG, Horowitz M, Jones KL. Postprandial hypotension: a systematic review. J Am Med Dir Assoc. 2014;15:394-409.

3. Walter BL. Cardiovascular autonomic dysfunction in patients with movement disorders. Clevel Clin J Med. 2008;75:S54-8.

4. Borg MJ, Jones KL, Sun Z, Horowitz M, Rayner CK, Wu T. Metformin attenuates the postprandial fall in blood pressure in type 2 diabetes. Diabetes Obes Metabol. 2019;21:1251-4.

5. Eschlbock S, Wenning G, Fanciulli A. Evidence-based treatment of neurogenic orthostatic hypotension and related symptoms. J Neural Transm. 2017;124:1567-605.

6. Zhang J, Guo L. Effectiveness of acarbose in treating elderly patients with diabetes with postprandial hypotension. J Invest Med. 2017;65:772-83.

7. Ong AC, Myint PK, Potter JF. Pharmacological treatment of postprandial reductions in blood pressure: a systematic review. J Am Geriatr Soc. 2014;62:649-61.

8. Biering-Sorensen F, Biering-Sorensen T, Liu N, Malmqvist L, Wecht JM, Krassioukov A. Alterations in cardiac autonomic control in spinal cord injury. Auton Neurosci. 2018;209:4-18.

9. Partida E, Mironets E, Hou S, Tom VJ. Cardiovascular dysfunction following spinal cord injury. Neural Regen Res. 2016;11:189-94.

10. Ishikawa J, Watanabe S, Harada K. Awakening blood pressure rise in a patient with spinal cord injury. Am J Case Rep. 2016;17:177-81.

11. Catz A, Mendelson L, Solzi P. Symptomatic postprandial hypotension in high paraplegia. Case Rep Paraplegia. 1992;30:582-6.

12. Catz A, Bluvshtein V, Pinhas I, Akselrod S, Gelernter I, Nissel T, et al. Hemodynamic effects of liquid food ingestion in midthoracic paraplegia: is supine postprandial hypotension related to thoracic spinal cord damage? Spinal Cord. 2007;45:96-103.

13. Jian ZJ, Zhou BY. Efficacy and safety of acarbose in the treatment of elderly patients with postprandial hypotension. Chin Med J. 2008;121:2054-9.

14. Bauman WA, Spungen AM. Disorders of carbohydrate and lipid metabolism in veterans with paraplegia or quadriplegia: a model of premature aging. Metabolism. 1994;43:749-56.

15. Bauman WA, Spungen AM. Risk assessment for coronary heart disease in a veteran population with spinal cord injury. Top Spinal Cord Inj Rehabil. 2007;12:35-53. 
16. Duckworth WC, Solomon SS, Jallepalli P, Heckemeyer C, Finnern J, Powers A. Glucose intolerance due to insulin resistance in patients with spinal cord injuries. Diabetes. 1980;29:906-10.

17. Duckworth WC, Jallepalli P, Solomon SS. Glucose intolerance in spinal cord injury. Arch Phys Med Rehabil. 1983;64:107-10.

18. Stillman M, Graves D, Lenneman C, Williams S. Neurogenic bowel, disordered glycemic control and chronic spinal cord injury: a preliminary investigation. Phys Med Rehabil - Int. 2017;4:1-3.

19. Kearney MT, Cowley AJ, Stubbs TA, Macdonald IA. Effect of a physiological insulin infusion on the cardiovascular responses to a high fat meal: evidence supporting a role for insulin in modulating postprandial cardiovascular homoeostasis in man. Clin Sci. 1996;91:415-23.

20. Kearney MT, Cowley AJ, Stubbs TA, Evans A, Macdonald IA. Depressor action of insulin on skeletal muscle vasculature: a novel mechanism for postprandial hypotension in the elderly. J Am Coll Cardiol. 1998;31:209-16.

21. Sasaki E, Kitaoka H, Ohsawa N. Postprandial hypotension in patients with non-insulin-dependent diabetes mellitus. Diabetes Res Clin Pract. 1992;18:113-21.

22. Jones KL, Horowitz M, Carney BI, Wishart JM, Guha S, Green L. Gastric emptying in early noninsulin-dependent diabetes mellitus. J Nucl Med. 1996;37:1643-8. 